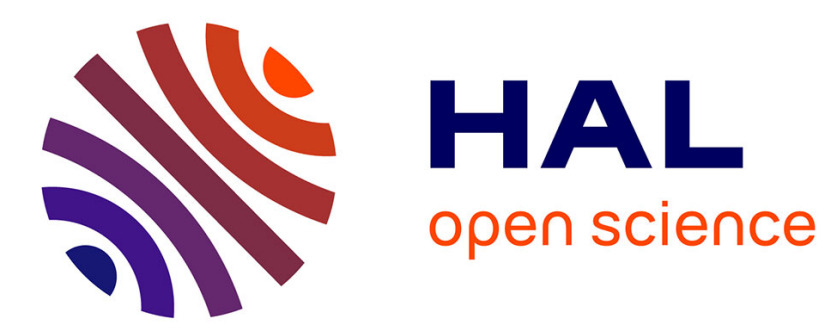

\title{
Long cycles in fullerene graphs
}

Rok Erman, František Kardoš, Jozef Miškuf

\section{To cite this version:}

Rok Erman, František Kardoš, Jozef Miškuf. Long cycles in fullerene graphs. Journal of Mathematical Chemistry, 2009, 46 (4), pp.1103-1111. 10.1007/s10910-008-9495-z . hal-00966741

\section{HAL Id: hal-00966741 https://hal.science/hal-00966741}

Submitted on 27 Mar 2014

HAL is a multi-disciplinary open access archive for the deposit and dissemination of scientific research documents, whether they are published or not. The documents may come from teaching and research institutions in France or abroad, or from public or private research centers.
L'archive ouverte pluridisciplinaire HAL, est destinée au dépôt et à la diffusion de documents scientifiques de niveau recherche, publiés ou non, émanant des établissements d'enseignement et de recherche français ou étrangers, des laboratoires publics ou privés. 


\title{
Long cycles in fullerene graphs
}

\author{
Rok Erman ${ }^{a *}$ František Kardoš ${ }^{b \dagger}$ and Jozef Miškuf ${ }^{b \ddagger}$ \\ ${ }^{a}$ Department of Mathematics, University of Ljubljana, \\ Jadranska 19, 1111 Ljubljana, Slovenia \\ rok.erman@gmail.com \\ ${ }^{b}$ Institute of Mathematics, Faculty of Science, P. J. Šafárik University, \\ Jesenná 5, 04001 Košice, Slovakia \\ \{frantisek.kardos, jozef.miskuf\}@upjs.sk
}

\begin{abstract}
It is conjectured that every fullerene graph is hamiltonian. Jendrol' and Owens proved [J. Math. Chem. 18 (1995), pp. 83-90] that every fullerene graph on $n$ vertices has a cycle of length at least $4 n / 5$. Recently, Král et al. improved it to $5 n / 6-2 / 3$.

In this paper, we study 2 -factors of fullerene graphs. As a by-product, we get an improvement of a lower bound on the length of the longest cycle in a fullerene graph. We present a constructive proof of the bound $6 n / 7+2 / 7$.
\end{abstract}

\section{Introduction}

Since the discovery of the first fullerene molecule [13] in 1985, the fullerenes have been objects of interest to scientists all over the world. The name fullerenes was given to cubic carbon molecules in which the atoms are arranged on a sphere in pentagons and hexagons. Fullerene graphs were defined as cubic (i.e. 3-regular) planar 3-connected graphs with pentagonal and hexagonal faces.

Various structural properties of fullerene graphs have been studied. See the papers $[1,2,3,18]$ for results on perfect matchings i.e. Kekulé structures of fullerenes. In [8] the independence number of fullerenes is studied. See [14] for a list of open problems on fullerene graphs. One of the central questions remains to see whether this class of graphs is hamiltonian.

The hamiltonicity of various subclasses of 3-connected planar cubic graphs has been investigated. Grünbaum and Zaks [9] asked whether the graphs in the family $\mathcal{G}_{3}(p, q)$

\footnotetext{
*Supported in part by bilateral project BI-SK/05-07-001 between Slovenia and Slovakia.

${ }^{\dagger}$ Supported in part by bilateral project SK-SI-00806 between Slovakia and Slovenia.

${ }^{\ddagger}$ Supported in part by Slovak Research and Development Agency under the contract No. APVV0007-07.
} 
of 3-connected cubic planar graphs whose faces are of size $p$ and $q$ with $p<q$ are hamiltonian for any $p, q$. Note that $p \in\{3,4,5\}$ by Euler's formula. For recent results see $[6,7,16,17]$.

Let us restrict our attention to fullerene graphs. For every fullerene graph, the leapfrog fullerene (graph obtained by replacing each vertex by a hexagon, see e.g. [5] for details) contains a hamiltonian path [15]. In general, Ewald [4] proved that every cubic planar graph graph with faces of size at most 6 contains a cycle which meets every face of $G$. This implies that there is a cycle through at least $n / 3$ of the vertices of any fullerene graph on $n$ vertices. Jendrol' and Owens [10] gave a better bound of $4 n / 5$. Recently, Král et al. [12] improved it to $5 n / 6-2 / 3$.

\section{Results}

The main result can be stated in the following way.

Theorem 1 Let $G$ be a fullerene graph on $n>380$ vertices. The length of the longest cycle in $G$ is at least $\frac{6 n+2}{7}$.

We will prove the following propositions, from which the statement of Theorem 1 immediately follows.

Proposition 1 Let $G$ be a fullerene graph on $n>380$ vertices. There is a 2-factor of $G$ consisting of at most $\frac{n+12}{14}$ cycles.

Proposition 2 Let $G$ be a fullerene graph on $n$ vertices. If there is a 2-factor $G^{\prime}$ consisting of c cycles, then there is a cycle in $G$ of length at least $n-2(c-1)$.

\section{Proof of Proposition 1}

Let us start with several definitions.

A perfect matching in a graph $G$ is a set of edges such that every vertex of $G$ is incident with precisely one of them. A $k$-factor in a graph $G$ is a $k$-regular spanning subgraph of $G$. A 1-factor is just a perfect matching. Note that if $M$ is a perfect matching of a cubic graph $G$, then $E(G) \backslash M$ forms a 2-factor of $G$.

Let $G$ be a fullerene graph and $M$ be a perfect matching in $G$. A hexagonal face $f$ is called resonant in $M$, if precisely three edges incident with $f$ are in $M$. Let $G$ be a fullerene graph and $G^{\prime}$ be a 2-factor in $G$. A hexagonal face $f$ is called a shift, if precisely three non-adjacent edges incident with $f$ are in $G^{\prime}$. It is easy to see that $f$ is resonant in $M$ if and only if $f$ is a shift in $E(G) \backslash M$.

The idea of the proof is as follows: First, we find a 2-factor in $G$. We proceed to delete some edges and after that add some other edges, in such a way that we get a collection of long cycles. 


\subsection{Search for a good start}

Lemma 1 Let $G$ be a fullerene graph on $n>380$ vertices. Then there is a 2-factor $G^{\prime}$ of $G$ such that at least one hexagon is a shift.

Proof. In [11], it was proven that for every fullerene graph $G$ on $n$ vertices there is a decomposition of its edges into three perfect matchings $M_{1}, M_{2}, M_{3}$, such that at least one of them, say $M_{1}$, has at least $\frac{n-380}{61}$ resonant hexagons. Then $G^{\prime}=M_{2} \cup M_{3}$ is a 2 -factor of the graph $G$ having at least $\frac{n-380}{61}$ shifts.

Let the edges of $G^{\prime}$ be black and the rest be white.

Lemma 2 There are no three shifts $f_{1}, f_{2}, f_{3}$ incident with a common vertex $v$.

Proof. Let $v$ be a vertex of $G$. Then precisely two of the three edges incident with $v$ are black and one of them is white. Thus, the face incident with the two black edges can not be a shift.

Let us transform all shifts as follows: For each shift $f$, recolor all three black edges incident with $f$ white. Insert a new vertex $v_{f}$ in the center of $f$ and join $v_{f}$ with the six vertices incident with $f$ by six new black edges. Let the configuration of six triangles obtained this way be called a hole. Repeat the procedure until there are no shifts left. Let the resulting plane graph be $G_{1}$ (see Figure 1).

If we delete all the white edges in $G_{1}$, we get the graph $G_{2}$. Each vertex has degree 2 or 6 in it. We introduce a plane pseudograph (a graph with multiple edges and loops) $H$ derived from $G_{2}$ by the following operation:

Contract every non-loop edge with at least one end-vertex of degree 2. Repeat this step until there are no more edges of this type. Note that the resulting pseudograph $H$ does not depend on the order of the contractions. Its components are either loops, or 6-regular connected plane pseudographs. Vertices of degree 6 in $H$ correspond to holes in $G_{1}$.
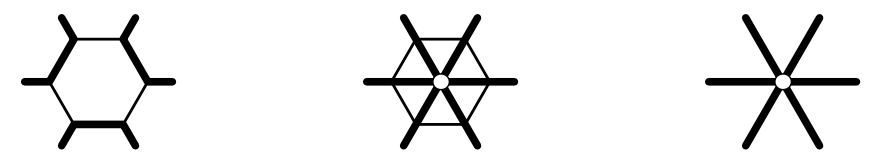

Figure 1: A shift in $G$, a hole in $G_{1}$ and a corresponding structure of $H$.

A loop $L$ in $H$ forming a component corresponds to a black cycle $C$ in $G$. Recolor the (black) edges on cycles of $G$ corresponding to such loops of $H$ blue.

Lemma 3 There are no blue cycles in $G$.

Proof. Assume there is at least one blue cycle in $G$. Since there is at least one shift in $G$, there is at least one black cycle in $G$. Therefore, there must be a face $f$ incident with a blue edge and a black edge. Let $C$ be the blue cycle incident with $f$ and $C^{\prime}$ be the 


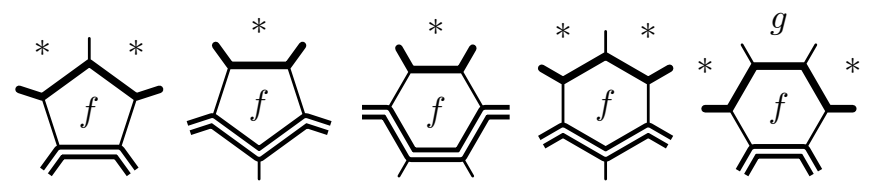

Figure 2: The list of all possible positions of black and blue edges incident with a face $f$. Blue edges are doubled.

black cycle incident with $f$. There are five possibilities (up to symmetry) of the position of blue and black edges incident with $f$, see Figure 2 .

Since $C$ is not adjacent to a hole in $G_{1}$, faces marked with an asterisk can not become holes, for they are incident with two consecutive black edges. If the face $g$ were to become a hole in the process of obtaining $G_{1}$, it forces the face $f$ to become a hole (see Figure 3). A contradiction with $C$ not being adjacent to a hole in $G_{1}$. Therefore $C^{\prime}$ is a cycle not adjacent to any hole in $G_{1}$ thus it is blue, which is a contradiction.

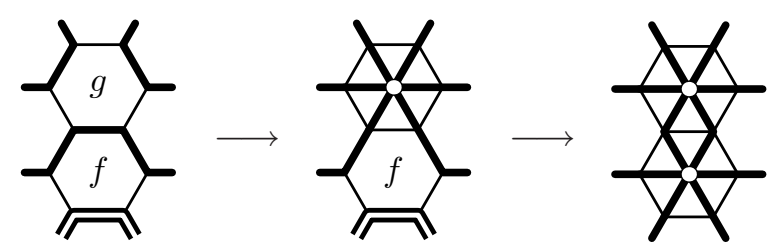

Figure 3: Left to right: the face $f$ incident with a blue cycle and a black cycle, adjacent to a shift $g$ in $G$; the face $f$ incident with a blue cycle adjacent to a hole, $f$ is now a shift; the face $f$ becomes a new hole. Blue edges are doubled.

After this step there are no isolated loops in the graph $H$. There still can be some loops incident with a vertex of degree six (corresponding to a hole of $G_{1}$ ).

Lemma 4 If there are loops in $H$, then there are only two black cycles in $G$.

Proof. Lemma 3 insures that there are no blue cycles in $G_{1}$. Therefore, the graph $H$ is 6-regular.
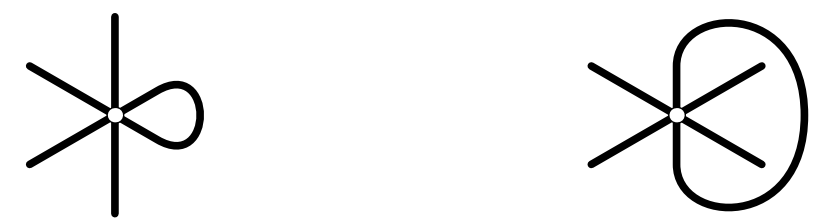

Figure 4: The two possible types of loops incident with a vertex of $H$.

Let the six edge ends incident with a vertex $f$ in $H$ be denoted by $1, \ldots, 6$. Let us suppose that there is a loop $l$ at $f$. It is obvious that it can connect two adjacent edge ends or two opposite ones. Without loss of generality it can either connect 1 and 2 or 3 and 6 . If it connects 1 and 2 as in Figure 5, since there are no other holes incident 


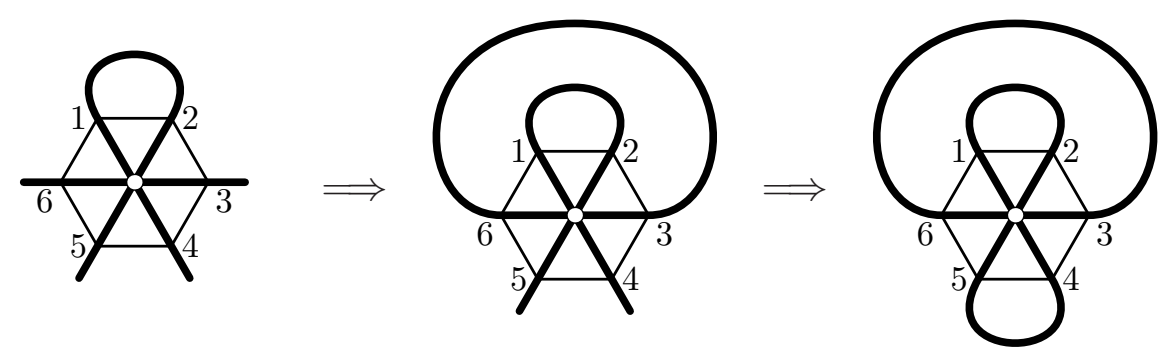

Figure 5: One loop forces other two.

with any edge of the black cycle in $G_{1}$ corresponding to the loop $l$, we apply the same reasoning as in Lemma 3 to come to the conclusion that there is a loop connecting 3 and 6 and after one more step a loop connecting 4 and 5 .

If it connects 3 and 6 as in Figure 4, we simply apply the reasoning from Lemma 3 to the loop connecting 3 and 6 to get a loop connecting 1 and 2 and a loop connecting 4 and 5 (see Figure 5).

Therefore the only configuration of a hole with a loop is the rightmost one in Figure 5.

In this case there are no other holes adjacent to the loops, which means that the three loops contain all vertices of $G_{1}$. This means that $f$ was the only shift in $G$ and therefore there are only two black cycles in $G$.

In the following we deal only with the case where there are no loops in $H$.

\subsection{Deciding the holes}

Now, we have the graph $G$ in which black edges form a 2 -factor, and the graph $G_{1}$ in which shifts were changed into holes. In $G_{1}$, the black edges form a set of paths going from one hole to another. Let us call these paths tracks.

We introduce an algorithm for deciding the holes in such a way that all resulting black cycles are long enough. By deciding a hole $f$ we mean recoloring three non-adjacent edges incident with $f$ black and removing the vertex $v_{f}$ - this way the hole is changed back to a shift. There are always two possible decisions for each hole.

At the beginning, all black edges are on tracks. During the process, some black cycles and closed tracks will be created. By closed track we mean track that emerge and end in the same hole. Let us define the inside of a closed track $\ell$ : Let $C$ be the component of $G \backslash \ell$ that has less vertices. Then the inside of $\ell$ is the graph induced on $C \cup \ell$. If we create a closed track such that there are less than three faces inside it, we color it red. We show that it is possible to find a sequence of hole decisions such that the number of red closed tracks does not exceed one.

If there is a red closed track $\ell$, we decide the hole $f$ incident with it in the way that prevents us from creating a short cycle, see Figure 6 . We show that there is at most one new red closed track.

Let us assume there are two new red closed tracks. Then the two tracks incident with $f$ are parts of two distinct red closed tracks. The closed track containing the edges 


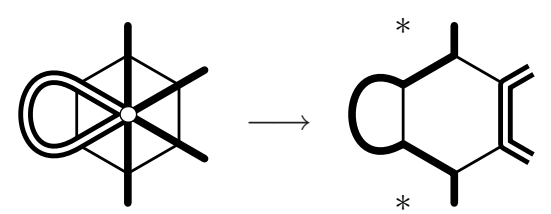

Figure 6: Deciding the hole incident with a red closed track. Edges of the red closed track are doubled.

of $\ell$ contains at least the two faces adjacent to $f$ (marked with an asterisk in Figure 6). The track can only be red if these are the only faces inside it. But that is not possible, since the two faces can not be adjacent in a fullerene graph.

Let there be no red closed tracks.

Let us define three special types of holes. A hole $f$ is of type $a$, if all tracks starting in $f$ end in the same hole $f^{\prime}$. A hole $f$ is of type $b$, if precisely 5 of the tracks starting in $f$ end in the same hole $f^{\prime}$. A hole $f$ is of type $c$, if three tracks starting in $f$ end in $f^{\prime}$ and the other three end in $f^{\prime \prime} \neq f^{\prime}$.

The holes of the type $a$ appear only in pairs. We can always decide those pairs in such a way that we get a single cycle, and the length of such cycle is at least 24 , see Figure 7.
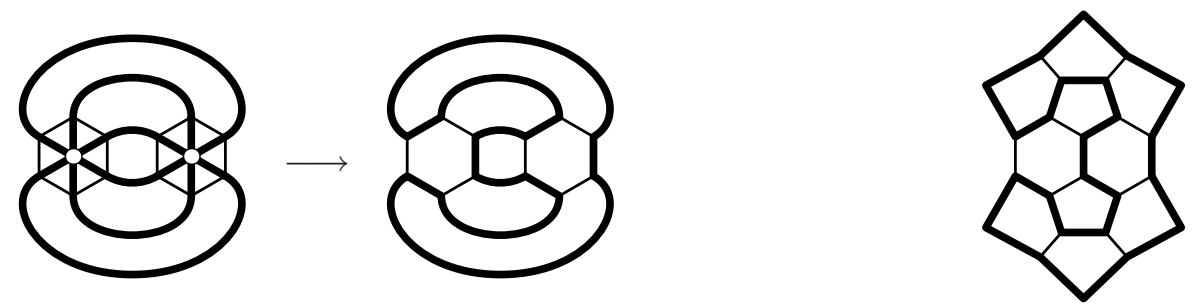

Figure 7: Deciding the pair of holes of the type $a$; the shortest cycle that can be obtained this way.

The holes of the type $b$ appear only in pairs, too. We can always decide those pairs in such a way that we get a long track. If the obtained track is closed its length is at least 19 edges (see Figure 8) and it is never red.
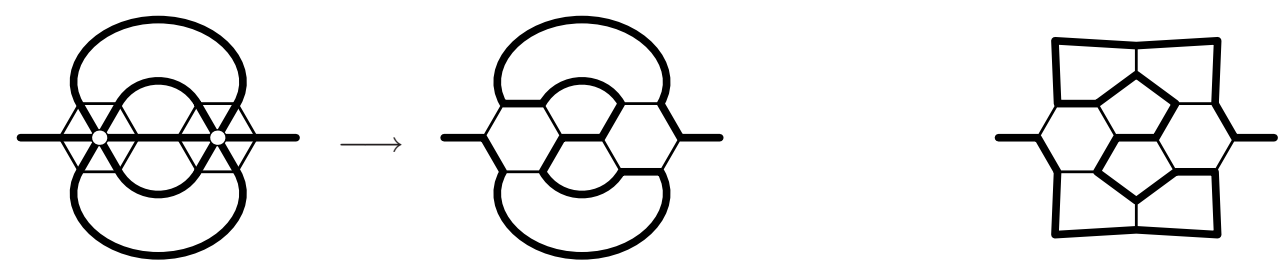

Figure 8: Deciding the pair of holes of the type $b$; the shortest track that can be obtained this way.

Let there be no holes of the types $a$ or $b$.

Let $f$ be a hole. There are two possible decisions for it. We claim that if $f$ is not of type $c$, it can be decided in such a way that we get at most one red closed track. 
For sake of a contradiction, assume that both decisions lead to at least two red closed tracks. Mark the tracks ending in $f$ as $p_{1}$ up to $p_{6}$ in cyclic order. One decisions joins $p_{1}$ with $p_{2}, p_{3}$ with $p_{4}$ and $p_{5}$ with $p_{6}$. Without loss on generality suppose that $p_{1}$ with $p_{2}$ and $p_{3}$ with $p_{4}$ form two closed tracks. It means that $p_{1}$ and $p_{2}$ end in the same hole $f_{12}$ and $p_{3}$ and $p_{4}$ end in $f_{34}$. If $f_{12}=f_{34}$, then since $f$ is not of type $a$ or $b, p_{5}$ and $p_{6}$ do not end in that hole. Then the other decision can make only one closed track, hence only one red closed track indeed, a contradiction (see Figure 9 for illustration). If $f_{12} \neq f_{34}$, then we can only get two closed tracks for the other decision if $f$ is of type $c$, a contradiction again.

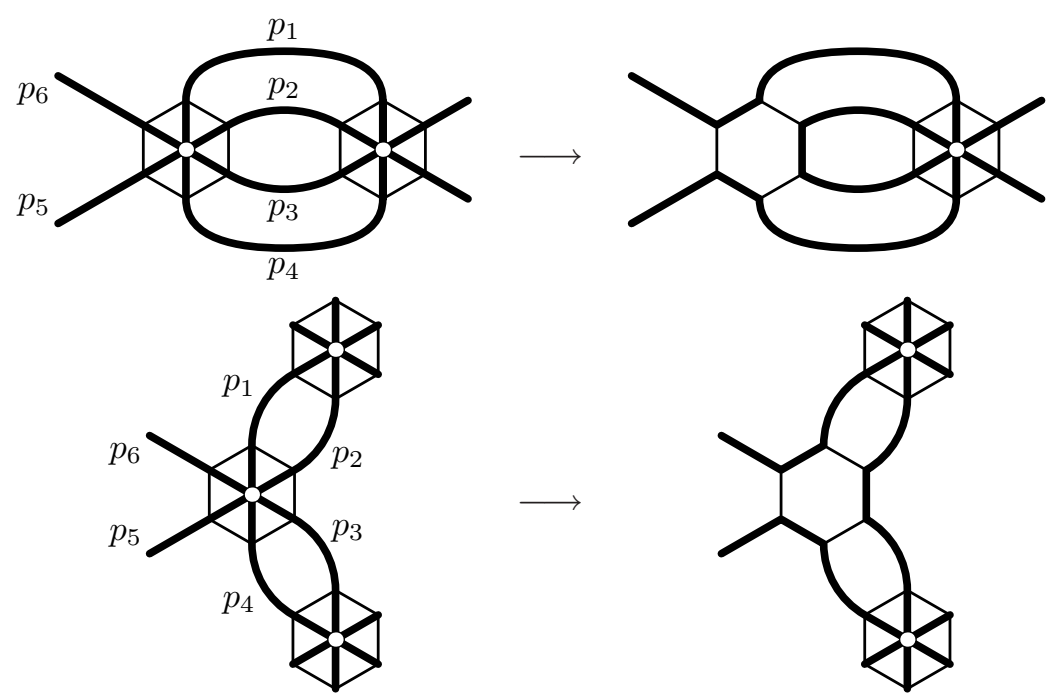

Figure 9: If the pairs of tracks $p_{1}, p_{2}$ and $p_{3}, p_{4}$ lead to closed tracks, the other decision of the hole leads to at most one red closed track.

If there are no red closed tracks and all holes are of the type $c$, the graph $H$ consists of cycles with tripled edges. Consider one of them. If the number of holes on the cycle is even, we can decide them in such a way that we get only one long black cycle, see Figure 10. If their number is odd, we can decide them in such a way that we get two cycles of big average length, see Figure 11.
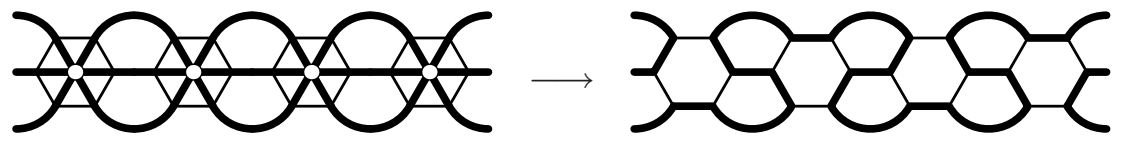

Figure 10: Deciding the cycle of holes of the type $c$.

Altogether, at the end of this process all the holes have been decided and black edges form a 2-factor again.

There are cycles of length at least 24 formed by pairs of holes of type $a$, pairs of cycles of average length at least 15 formed by holes of type $c$, or cycles with at least three faces inside. If there are three hexagons, the cycle has length 14. If there are $k$ pentagons and $3-k$ hexagons, the cycle has length $14-k$. 

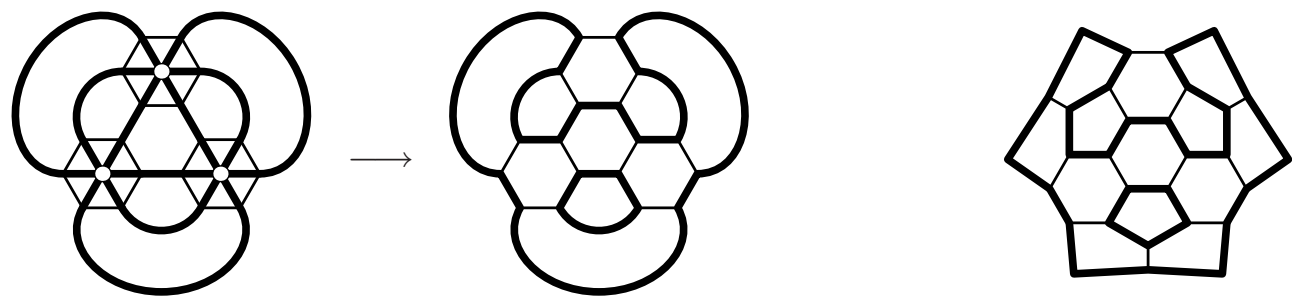

Figure 11: If the holes of the type $c$ form an odd cycle, we always get two cycles (left); the shortest possible pair of cycles that can be obtained this way have lengths 5 and 25 (right).

Let the number of cycles of length 11 be $c_{11}$, the number of cycles of length 12 be $c_{12}$, the number of cycles of length 13 be $c_{13}$, the number of all other cycles be $c_{14}$ and let $c=c_{11}+c_{12}+c_{13}+c_{14}$. Then since there are exactly 12 pentagons in a fullerene graph,

$$
3 c_{11}+2 c_{12}+c_{13} \leq 12 \text {. }
$$

On the other hand,

$$
11 c_{11}+12 c_{12}+13 c_{13}+14 c_{14} \leq n .
$$

Adding the two equations yields

$$
14 c \leq n+12
$$

This is the bound needed.

\section{Proof of Proposition 2}

Proof. Let $G^{\prime}$ be a 2-factor. We color the edges of $G^{\prime}$ black, the other edges white. Initially, we color all the vertices black and then we recolor the vertices not on any black cycle to white.

If there are at least two cycles in the graph $G^{\prime}$, there must be a face $f$ incident with two different cycles $C_{1}$ and $C_{2}$. If the face $f$ is a pentagon, then we recolor the black edges incident with $f$ white and vice-versa. This way the number of white vertices increases or decreases by 1 (see Figure 12 for illustration).

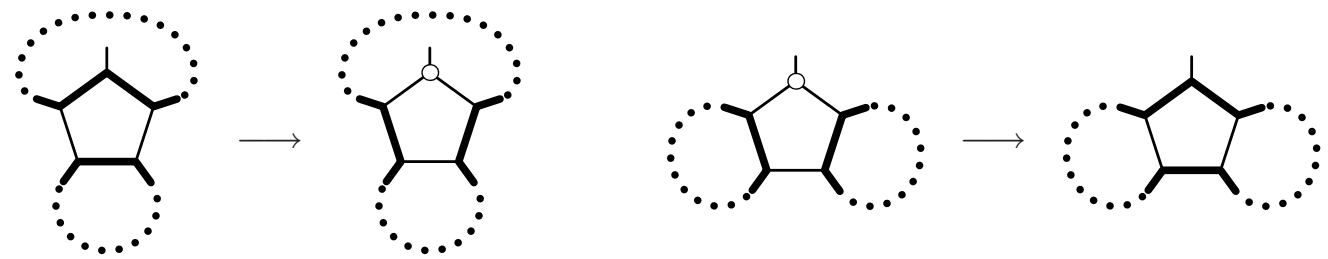

Figure 12: If a pentagon is incident with two cycles, we can change them to one cycle in such a way that the number of white vertices increases or decreases by 1 . 


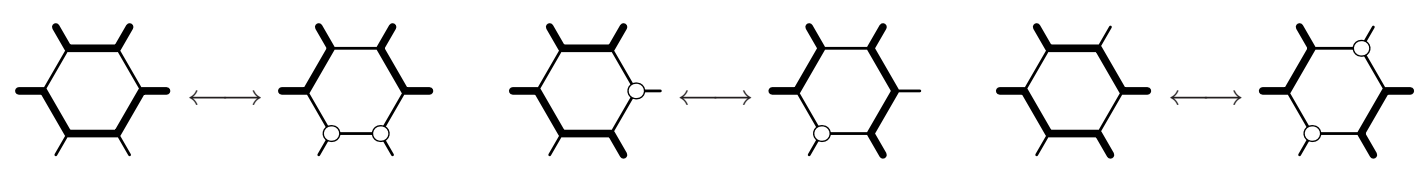

Figure 13: If a hexagon is incident with two cycles, we can change them to one cycle in such a way that the number of white vertices increases by at most 2 .

If the face $f$ is a hexagon which is not a shift, then the black edges incident with $f$ induce 2 paths. We recolor the black edges incident with $f$ white and vice-versa. This way the number of white vertices changes by $-2,0$, or 2 (see Figure 13 for illustration).

If the face $f$ is a shift, then two of its black edges are on one cycle, say $C_{1}$, and one on the other cycle, $C_{2}$. If any of the two faces adjacent to $f$ and incident both with $C_{1}$ and $C_{2}$ is not a shift, we use that face to join $C_{1}$ and $C_{2}$. If both those faces are shifts, then we change the color of the edges incident with the shift $f$. The number of cycles does not change, and the two faces that shared a black $C_{1}$ edge with $f$ can now be used to join $C_{1}$ and $C_{2}$, see Figure 14 .

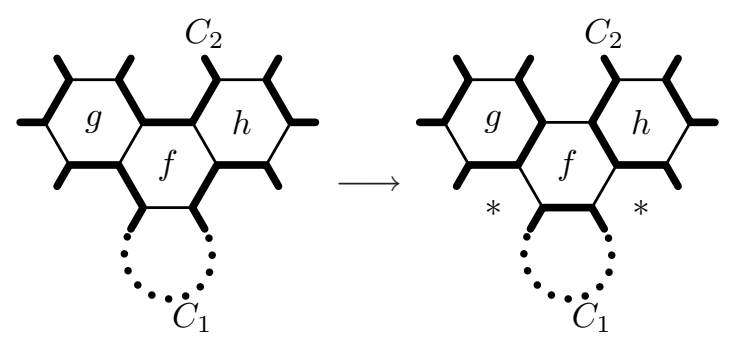

Figure 14: If a shift $f$ is incident with two cycles and $g$ and $h$ are shifts too, we can first switch the shift $f$ and then change any of the faces marked with an asterisk to change the cycles to one cycle.

This way we can always decrease the number of cycles by introducing at most 2 white vertices per cycle. Hence, the number of white vertices $w$ is at most $2(c-1)$.

\section{References}

[1] T. Došlić, On lower bounds of number of perfect matchings in fullerene graphs, J. Math. Chem. 24 (1998) 359-364.

[2] T. Došlić, On some structural properties of fullerene graphs, J. Math. Chem. 31 (2002) 187-195.

[3] T. Došlić, Saturation number of fullerene graphs, J. Math. Chem. 43 (2008) 647657.

[4] G. Ewald, On shortness exponents of families of graphs, Israel J. Math. 16 (1973) 53-61. 
[5] P. W. Fowler and D. E. Manolopoulos, An Atlas of Fullerenes, Oxford Univ. Press, Oxford, 1995.

[6] P. R. Goodey, A class of hamiltonian polytopes, (special issue dedicated to Paul Turán) J. Graph Theory 1 (1977) 181-185.

[7] P. R. Goodey, Hamiltonian circuits in polytopes with even sided faces Israel J. Math. 22 (1975) 52-56.

[8] J. Graver, The Independence Numbers of Fullerenes and Benzenoids, European J. Comb. 27 (2006) 850-863.

[9] B. Grünbaum, J. Zaks, The existence of certain planar maps, Discrete Math. 10 (1974) 93-115.

[10] S. Jendrol' and P. J. Owens, Longest cycles in generalized Buckminsterfullerene graphs, J. Math. Chem. 18 (1995) 83-90.

[11] F. Kardoš, D. Král, J. Miškuf, J.-S. Sereni, Fullerene graphs have exponentially many perfect matchings, arXiv:0801.1438v1 [math.CO].

[12] D. Král, O. Pangrác, J.-S. Sereni, R. Škrekovski, Long cycles in fullerene graphs, J. Math. Chem. (online), doi: 10.1007/s10910-008-9390-7.

[13] H. W. Kroto, J. R. Heath, S. C. O'Brien, R. F. Curl and R. E. Smalley, $C_{60}$ Buckminsterfullerene, Nature 318 (1985) 162-163.

[14] J. Malkevitch, Geometrical and Combinatorial Questions About Fullerenes, in: P. Hansen, P. Fowler, M. Zheng (Eds.), Discrete Mathematical Chemistry, DIMACS Series in Discrete Mathematics and Theoretical Computer Science 51 (2000) 261-166.

[15] D. Marušič, Hamilton Cycles and Paths in Fullerenes, J. Chem. Inf. Model., 47 (3) (2007), 732-736.

[16] P. J. Owens, Non-hamiltonian simple 3-polytopes with only one type of face besides triangles, Ann. Discrete Math. 20 (1984), 241-251.

[17] J. Zaks, Non-hamiltonian simple 3-polytopes having just two types of faces, Discrete Math. 29 (1980) 87-101.

[18] H. Zhang and F. Zhang, New lower bound on the number of perfect matchings in fullerene graphs, J. Math. Chem. 30 (2001) 343-347. 